\title{
Analysis of Effects on Dynamic Alignment Precision of Warship Combat System caused by Its Attitude
}

\author{
Zhiheng Zhou*, Jianjun Zhao, Deyi Sang and Libin Yang \\ Naval Aeronautical Engineering Institute, Department of Ordnance Science and Technology, Yantai, China, 264001; \\ ${ }^{*}$ Corresponding author
}

\begin{abstract}
According to warship combat system dynamic alignment demand, a coordinate system aligned combat system was established in this paper, the mainly coordinate conversion were derived, sensor measurement errors caused by warship's attitude were calculated and simulated by computer. Simulation results show that: the warship's heading angle causes the azimuth angle error of sensor measurements, the warship's pitch angle, roll angle cause the angle azimuth and elevation angle error of sensor measurements, and there is almost no influence on sensor distance measurement caused by warship's attitude.
\end{abstract}

Keywords-combat system; alignment in dynamic state; warship attitude; coordinate conversion; error analysis

\section{INTRODUCTION}

The target of warship combat system alignment is to make the machinery zero and electrical zero of all the systems such as detectors, trackers, weapon systems and navigation equipment all over the warship unified in the coordinate of deck system. It is to ensure the consistency of combat system zero position[1], and to ensure the consistent accordance of distance, azimuth/side angle, pitch angle about the same target by the identification of all the sensors in combat system[2]

Considering the dynamic environment of warship's mooring at dock or anchoring at sea, the sensors are required to measure the azimuth, elevation angle and distance of the calibration object at sea in the process of alignment, warship attitude changes will inevitably lead sensor deviation for each target observations, and then affect the alignment precision of combat system.

Currently, there are research results of static alignment, and has been well applicated in practical work[3,4].But there are almost no research on warship combat system alignment method and technology in dynamic state. Qi Cui[5], Xi Ma [6]have made some attempts, but those are only some improvement based on static alignment method, which did not consider the impact caused by warship's attitude to sensor measurement accuracy fully, and the sensor data are read artificial instantaneous instead of dynamic conditions as pseudo-dynamic alignment Literature[7-10] studied the impact on shipborne radar measurement accuracy caused by ship's swing, but they failed to understand the actual physical meaning of heading angle, pitch angle and roll angle of the of the platform compass under the Cartan ring frame structure, the coordinate transformation are inconsistent with the actual derivation.

\section{MAIN COORDINATE SYSTEM AND COORDINATE CONVERSION}

The sensors in warship combat system are in different location, with different target measurement elements, and they are also in different coordinate systems. In the process of alignment, we have to transform both the measurement data of each sensor and the true value which is acquired by true value acquisition device into the unified coordinate of deck system.

\section{A. Common Coordinate System}

\section{1) $E C E F$}

The origin $O_{e}$ is located in the center of the Earth's mass, and $X_{e}$ axis points at the intersection of equatorial plane and prime meridian, $Z_{e}$ axis coincides with the axis of rotation of the reference ellipsoid, pointing to Earth Arctic, $Y_{e}$ axis constitutes a right-handed Cartesian coordinate system with $X_{e}$ axis and $Z_{e}$ axis in the equatorial plane. A point in the coordinate system is indicated with $\left(x_{e}, y_{e}, z_{e}\right)$.

\section{2) Warship geographic coordinate system}

The origin $O_{l}$ of warship geographic coordinate system is located in the warship rocking center, $X_{l}$ axis parallels to the local horizontal plane and points true east, $Y_{l}$ parallels to the local horizontal plane and points true north, $Z_{l}$ axis perpendicular to the horizontal plane, $X_{l}, Y_{l}$ and $Z_{l}$ constitute a right-handed Cartesian coordinate system. Warship geographic coordinate system is the Cartesian coordinate system with the ship rocking center as origin. A point in the coordinate system is indicated with $\left(x_{l}, y_{l}, z_{l}\right)$.

\section{3) Warship coordinate of deck system}

The origin $O_{d}$ is located in the warship rocking center, $Y_{d}$ axis parallels to the ship bow and stern line, pointing bow is positive, $X_{d}$ axis perpendiculars to the $Y_{d}$ axis and parallels to the deck, pointing to the starboard side is positive, $Z_{d}$ axis perpendiculars to the deck up. A point in the coordinate system is indicated with $\left(x_{d}, y_{d}, z_{d}\right)$. 


\section{B. The definition of warship attitude angle}

Warship attitude angle were gestured by the gyrocompass, including the ship's heading, pitch and roll. Gyrocompass uses Cartan ring frame structure, and the diagram of its heading angle, pitch angle and roll angle are shown in Figure 1. In the figure, $O$ is the war ship rocking center, BCDEFNG is the plane, $M H J K L$ is the deck level, $J L$ is the vertical axis of the ship bow and stern line, and $A$ is the zenith. $O E$ is $X_{l}$ axis pointing east, $O N$ is $Y_{l}$ axis pointing north, $O A$ is $Z_{l}$ axis pointing to the zenith, $O E 、 O N$ and $O A$ constitute ships geographic coordinate system. The horizontal $O H$ is $X_{d}$ axis pointing to starboard, and the vertical axis is $Y_{d}$ axis pointing bow, and $O S$ is $Z_{d}$ axis perpendicular to $O H$ and $O J$, and constitute warship coordinate of deck system.

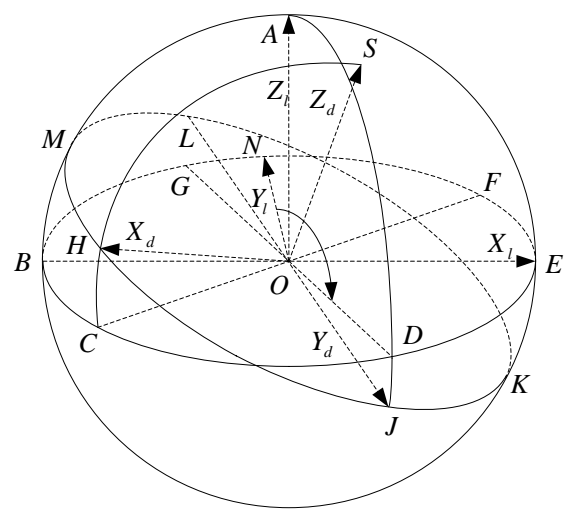

FIGURE I. WARSHIP ATTITUDE ANGLE SCHEMATIC PROGRESS

Heading angle $C_{w}$ indicates the clockwise rotation angle from the north to the direction of the ship's bow, measured in the horizontal plane, In the figure, and angle between $O N$ and $O D$ (projection of $O J$ in horizontal plane) is the heading angle, clockwise is positive.

Pitch angle $\psi$ indicates the rotated angle around the horizontal axis $\mathrm{OH}$ in the deck plane, that is the angle between the ship bow and stern lines and the horizontal plane, measured in the vertical plane. In the figure, arc $D J$ is the pitch angle, and bow over the horizontal plane is positive.

Roll angle $\theta$ indicates the rotated angle around the ship bow and stern line in deck level, that is the angle between $O C$ (the intersection line of warship's cross section and horizontal plane) and $\mathrm{OH}$ (the horizontal axis of the warship), the measurement plane perpendiculars to the deck level and perpendicular to the bow and stern line. In the figure, arc $H C$ is the roll angle, the right chord under pour is positive.

\section{The Main Conversion}

$$
\text { 1) }\left(x_{e}, y_{e}, z_{e}\right) \text { converts to }\left(x_{l}, y_{l}, z_{l}\right)
$$

For any point in space, when the origin of coordinate $\left(x_{e_{0}}, y_{e_{0}}, z_{e_{0}}\right)$ in warship geographic coordinate system and the latitude and longitude coordinates of the earth are known, we only need to translation origin of ECEF to the origin location of the warship geographic coordinate system first, and then make rotation transformation three times:

- First of all, rotates $\left(180+l_{0}\right)$ around $Z_{e}$ axis of ECEF ;

- Then rotates the gotten coordinate $\left(270+b_{0}\right)$ around $Y_{e}$ axis;

- Finally, rotates the gotten coordinate 180 degrees around $X_{e}$ axis.

According Bursa-Wolf model, the following formula can be obtained:

$$
\left[\begin{array}{c}
x_{l} \\
y_{l} \\
z_{l}
\end{array}\right]=T_{e l} \cdot\left[\begin{array}{c}
x_{e}-x_{e_{0}} \\
y_{e}-y_{e_{0}} \\
z_{e}-z_{e_{0}}
\end{array}\right]
$$

There into,

$$
\begin{aligned}
T_{e l} & =R_{X}\left(180^{\circ}\right) R_{Y}\left(B+270^{\circ}\right) R_{Z}\left(L+180^{\circ}\right) \\
& =\left[\begin{array}{ccc}
-\sin b_{0} \cos l_{0} & -\sin b_{0} \sin l_{0} & \cos b_{0} \\
-\sin l_{0} & \cos l_{0} & 0 \\
-\cos b_{0} \cos l_{0} & -\cos b_{0} \sin l_{0} & -\sin b_{0}
\end{array}\right]
\end{aligned}
$$

$$
\text { 2) }\left(x_{l}, y_{l}, z_{l}\right) \text { converts to }\left(x_{d}, y_{d}, z_{d}\right)
$$

The origin of ship geographic coordinate system coincides with the origin of the coordinate of deck system, so there is no translation transformation between them, but only rotation of angle. The reason of the rotation is the attitude changes of the warship, that is the warship's heading, pitch and roll. Since the ship gyrocompass use Cartan ring frame structure, Figure 2 shows that , from warship geographic coordinate system to the coordinate of deck system, first heading, then pitching and last rolling change must be ordered[11].That is, by heading transformation from $O-X_{l} Y_{l} Z_{l}$ (transformation matrix $R_{Z}\left(C_{w}\right)$--pivoting heading angle $C_{w}$ around $O Z_{l}$ ) to $O-X_{h} Y_{h} Z_{l}$, then by pitching transformation (transformation matrix $R_{X}(\psi)$--pivoting pitching angle $\psi$ around $O X_{h}$ ) to $O X_{h} Y_{d} Z^{\prime}$, and finally by rolling transformation (transformation matrix $R_{Y}(\theta)$--pivoting rolling angle $\theta$ around $O Y_{d}$ ) to $O_{d}-X_{d} Y_{d} Z_{d}[12]$. 


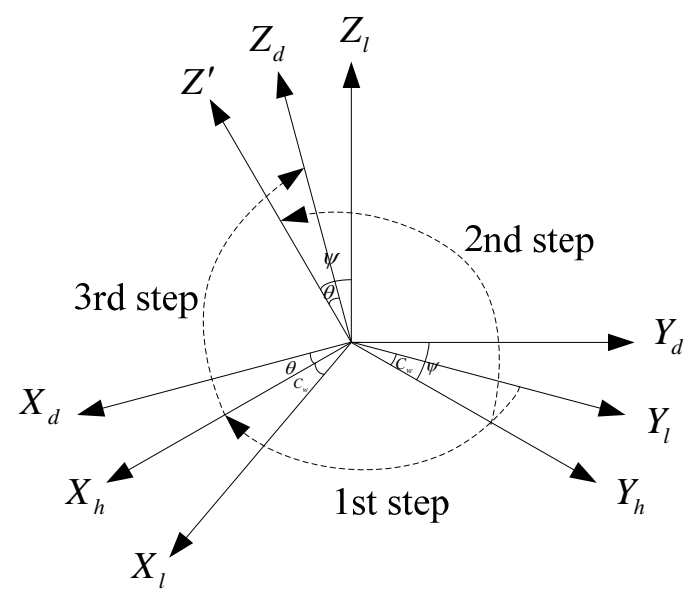

FIGURE II. TRANSFORMATION DIAGRAM

\section{INFLUENCE ANALYSIS OF DYNAMIC ALIGNMENT PRECISION CAUSED BY WARSHIP’s SWING}

Let us suppose that the warship is mooring in the dock or anchoring at sea, so the it is in a natural swing state because of the influence of wind and wave.

\section{A. Distance Error}

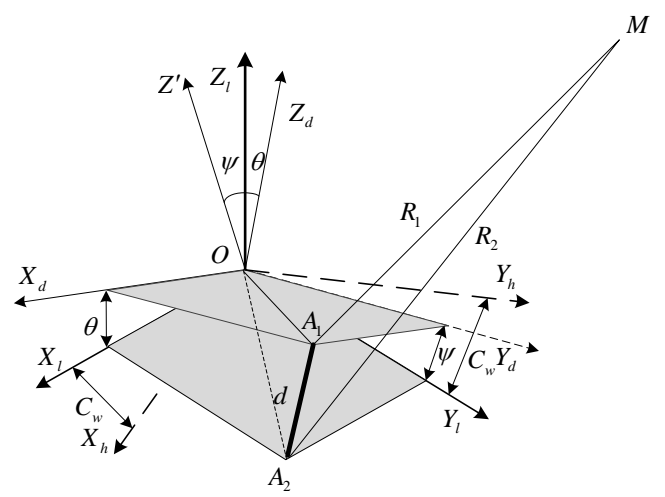

FIGURE III. SCHEMATIC DIAGRAM OF RADAR DISTANCE MEASUREMENT

Among them, heading transformation matrix is:

$$
R_{Z}(\gamma)=\left[\begin{array}{ccc}
\cos C_{w} & -\sin C_{w} & 0 \\
\sin C_{w} & \cos C_{w} & 0 \\
0 & 0 & 1
\end{array}\right]
$$

Pitching transformation matrix is:

$$
R_{X}(\psi)=\left[\begin{array}{ccc}
1 & 0 & 0 \\
0 & \cos \psi & \sin \psi \\
0 & -\sin \psi & \cos \psi
\end{array}\right]
$$

Rolling transformation matrix

$$
R_{Y}(\theta)=\left[\begin{array}{ccc}
\cos \theta & 0 & -\sin \theta \\
0 & 1 & 0 \\
\sin \theta & 0 & \cos \theta
\end{array}\right]
$$

Therefore, the rotation matrix

$$
T_{l d}=R_{Y}(\theta) R_{X}(\psi) R_{Z}\left(C_{w}\right)
$$

As is shown in Figure 3, the center of radar antenna is located at $A_{1}$, the target's distance measured by radar is $R_{1}$. When the ship's heading angle, pitch and roll angle, are respectively $C_{w}, \psi$ and $\theta$, the center of the radar antenna is located at the point $A_{2}$, the target's distance measured by radar is $R_{2}$, the displacement generated of the radar antenna center is $d$. Since the warship is in a slight swing, so the three attitude angle is very small, so $\angle A_{1} O A_{2}$ is small. Because the distance between warship rocking center and radar antenna center $O A_{1}=O A_{2}$ is not very big. so $d=2\left|O A_{1}\right| \sin \left(\angle A_{1} O A_{2} / 2\right)$ is small, much smaller than $R_{1}$ and $R_{2}$. Therefore, $\Delta R=\left|R_{1}-R_{2}\right|<d \approx 0$, so that the warship's rocking motion has little effect on radar distance measurement data.

\section{B. Angular error $\Delta E$ and $\Delta A$}

When the ship's heading angle, pitch angle and roll angle are respectively $C_{w}, \psi$ and $\theta$, and the plane $X_{d} O_{d} Y_{d}$ is not coincident with the plane $X_{1} O_{1} Y_{1}$, suppose that the angle between them is $\varphi$, then the angle between the normal vector of the two planes is also $\varphi$.

Get vector $\vec{a}=(0,0,1)$ in warship geographic coordinate system, then the vector is perpendicular to the plane $X_{1} O_{1} Y_{1}$, it can be considered as the normal vector of the plane, the vector is $\vec{a}^{\prime}$ after its conversion to coordinate of deck system, so $\vec{a}^{\prime}$ is the normal vector of the plane $X_{d} O_{d} Y_{d}$, then according to the formula:

$$
\begin{aligned}
\vec{a}^{\prime} & =T_{l d} \cdot \vec{a}=R_{Y}(\theta) R_{X}(\psi) R_{Z}\left(C_{w}\right) \cdot \vec{a} \\
& =\left[\begin{array}{c}
-\sin \theta \cos \psi \\
\sin \psi \\
\cos \theta \cos \psi
\end{array}\right]
\end{aligned}
$$

$\varphi$ is the angle between $\vec{a}^{\prime}$ and $\vec{a}$, because

$$
\vec{a}^{\prime} \cdot \vec{a}=\left|\vec{a}^{\prime}\right| \cdot|\vec{a}| \cdot \cos \varphi=\cos \theta \cos \psi
$$




$$
\varphi=\arccos (\cos \theta \cos \psi)
$$

Let the angle between $O X$ which is the projecting of normal vectors of plane $X_{d} O_{d} Y_{d}$ at plane $X_{l} O_{1} Y_{1}$ and $Y_{1}$ axis as $A_{m}$, can be determined by the ratio of $x_{l}$ and $y_{l}$ of the vector $\vec{a}^{\prime}$ in the plane $X O Y$.

$$
\tan A_{m}=-\sin \theta \cot \psi
$$

so

$$
A_{m}=-\arctan (\sin \theta \cot \psi)
$$

As shown in Figure 4, $Y$ axis is the pitch axis of radar antenna turntable, $Z$ axis is the azimuth axis of radar antenna turntable. When the warship rocks, radar antenna turntable is tilted, $Y_{d}$ and $Z_{d}$ is the pitch axis and azimuth axis of radar antenna turntable after it tilted. When the azimuth axis tilts, pitch axis rotates in the inclined surface.

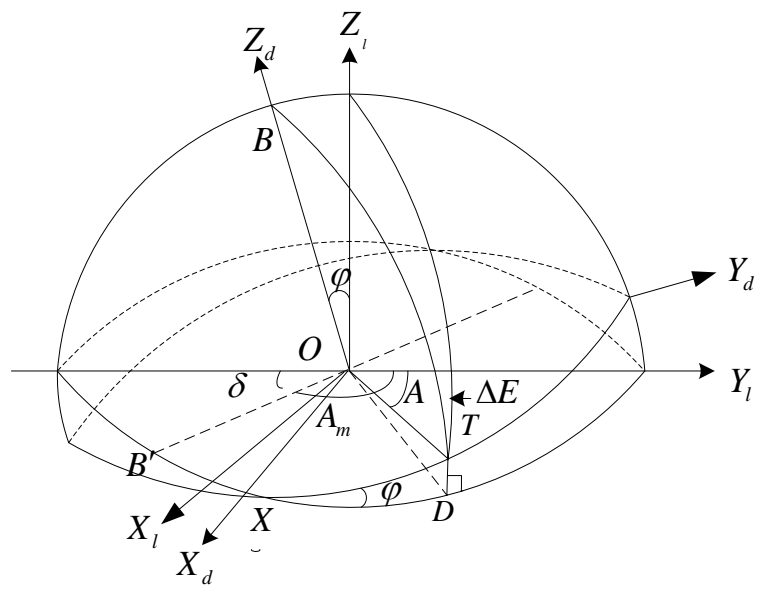

FIGURE IV. INFLUENCE ON ANGLE MEASUREMENT ACCURACY BY WARSHIP ATTITUDE

\section{4) Pitch angle error $\Delta E$}

When the pitch axis turns 0 degree, if the azimuth axis tilts, electronic axis is also in the inclined surface, and the angle between electronic axis and horizontal axis is the pitch angle error. When the orientation axis of rotates, the electronic axis is also rotates in the inclined surface, so the pitch error will change with the azimuth angle change. When the azimuth axis rotates to any azimuth $A$ the projector $O D$ in the horizontal plane of the electronic axis $O T$. In spherical right triangle $X T D$, there is

$$
\tan \Delta E=\tan \varphi \cos \left(A-A_{m}\right)
$$

Because $\varphi$ is small, $\tan \varphi \approx \varphi, \tan \Delta E \approx \Delta E$

So

$$
\Delta E=\varphi \cos \left(A-A_{m}\right)
$$

So

$$
\begin{aligned}
\Delta E & =\varphi \cos \left(A-A_{m}\right) \\
& =\arccos (\cos \theta \cos \psi) \cdot \cos (A+\arctan (\sin \theta \cot \psi))
\end{aligned}
$$

\section{5) Azimuth angle error $\Delta A$}

Because the pitch axis is perpendicular to electric axis, so the change rule of $\delta$ which is the pitch angle from pitch axis to level is 90 degree difference in the phase with $\Delta E$,

$$
\delta \approx \varphi \cdot \cos \left(\left(A-A_{m}\right)+90^{\circ}\right) \approx \varphi \cdot \sin \left(A-A_{m}\right)
$$

Due to pitch axis is non-horizontal, azimuth error caused by the rotation of pitch axis

$$
\Delta A \approx \delta \cdot \tan E
$$

Was substituted into the formula So

$$
\Delta A \approx \varphi \cdot \tan E \cdot \sin \left(A-A_{m}\right)
$$

Substituting will get into

$$
\begin{aligned}
& \Delta A \approx \varphi \cdot \tan E \cdot \sin \left(A-A_{m}\right)=\arccos (\cos \theta \cos \psi) \\
& \cdot \tan E \cdot \sin (A+\arctan (\sin \theta \cot \psi))
\end{aligned}
$$

\section{SimUlation ANALYSIS}

When warship is in the state of mooring at dock or anchoring at sea, alignment work is generally carried out when the weather is calm, warship's natural swing, and rotor helicopter equipped with a Luneberg lens reflector as the calibration object about $10 \mathrm{~km}$ away from the warship.

To study the impact on radar measurements caused by warship's attitude changes a number of simulation were conducted. The simulation results shown in Figure 5-7. 

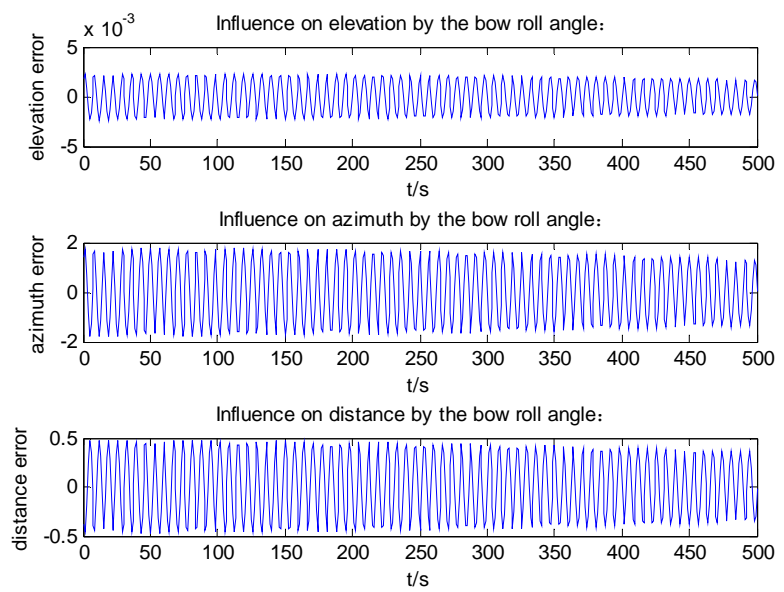

FIGURE V. INFLUENCE ON RADAR MEASUREMENT CAUSED BY THE BOW ROLL ANGLE

Figure 5 shows bow roll angle of the warship caused about $2 \mathrm{~m}$ distance error in the location information, caused about azimuth error, and the azimuth error has linear relationship with bow roll angle, while caused almost no effect on elevation.
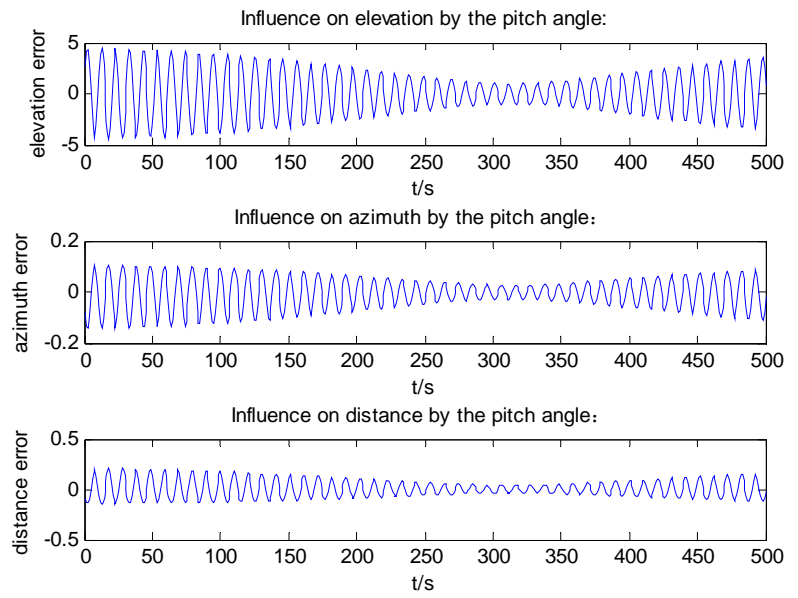

FIGURE VI. INFLUENCE ON RADAR MEASUREMENT CAUSED BY THE PITCH ANGLE

Figure 6 shows that of pitch angle the warship caused about $2 \mathrm{~m}$ distance error in the location information, caused about pitch angle error, and the elevation error has linear relationship with warship's pitch angle, while caused almost no effect on azimuth error.
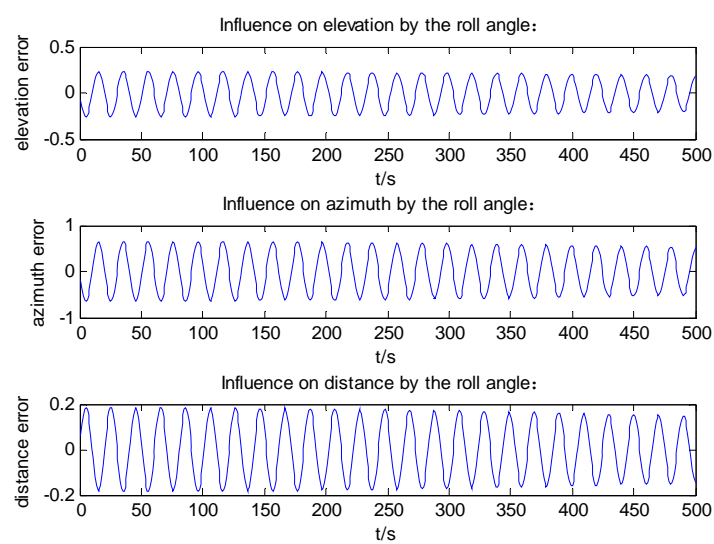

FIGURE VII. INFLUENCE ON RADAR MEASUREMENT CAUSED BY THE ROLL ANGLE.

Figure 7 shows roll angle of the warship caused about $0.5 \mathrm{~m}$ distance error in the location information, caused about in both elevation error and azimuth error, and the error curve has a highly correlated. with the ship rolling curve.
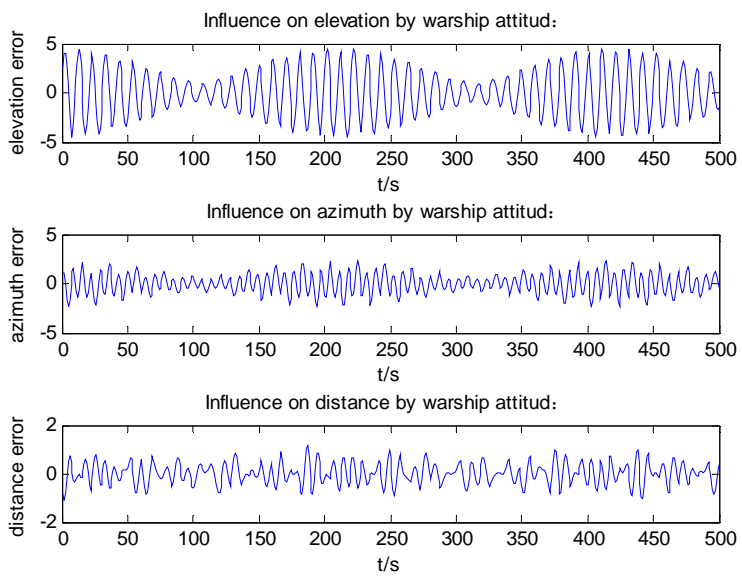

\section{FIGURE VIII. INFLUENCE ON RADAR MEASUREMENT CAUSED BY WARSHIP ATTITUDE}

Finally, considering the warship's bow roll angle, pitch angle and roll angle, the simulation was taken to calculate warship's attitude affect on warship radar measurement results, the simulation results was shown in Figure 8. It found that changes in warship's attitude caused about $5 \mathrm{~m}$ in distance error, in elevation error and in azimuth error.

\section{CONCLUSION}

Warship combat system alignment is the key work to ensure the warship's effectiveness operated fully, to ensure the weapon hit probability, and plays a fundamental and decisive role, therefore, alignment precision of the combat system is highly demanded. In this paper, the mainly coordinate conversion were derived, sensor measurement errors caused by warship's attitude were calculated and simulated by computer. Simulation results show that: the warship's heading angle 
causes the azimuth angle error of sensor measurements, the warship's pitch angle, roll angle cause the angle azimuth and elevation angle error of sensor measurements, and there is almost no influence on sensor distance measurement caused by warship's attitude. Therefore, in the subsequent data processing of dynamic alignment, sensor measurement errors need to be amended due to warship's attitude, in order to ensure alignment accuracy.

\section{REFERENCES}

[1] Xiadong Lv, Lei LI, Weilin LI, Xi MA.A Unified Radar Alignment Model with Error Analysis for Shipborne Combat Systems[J].Chinese Journal of Ship Research,2009,12(6):53-57.

[2] Zheng-hu Wang.Study on Control Accuracy of Alignment of Combat System in Ship Construction[D].Dalian:Dalian University of Technology,2014.

[3] Shizong Wei, Qin Zou.Within the shipboard combat system dock aligned explore [J]. Ship Engineering, 2001,12 (2): 12-14,23.

[4] Bowen Wang.Alignment Zero for Shipborne Combat Systems[J] .Naval Eqipment,2009,8:34-35.

[5] Qi CUI,Jianfei Zheng.Investigation of Dynamic Alignment Method for Shipborne Combat Systems[J].Ship Engineering Research,2010,12(4):29-33.

[6] Xi MA,Hao Feng,Xiadong Lv.A Method of Pre-estimating Theoretical Value for Shipborne Sensor System in Mooring State[J].Chinese Journal of Ship Research,2011,36(7):159-162.

[7] Shiyan Sun, Guodong Zhang.Target Tracking Error Analysis Caused by Ship's Pitch And Roll[J].Fire Control \& Command Control,2011,6(5):75-78.

[8] Xiaoqiang Wu, Lintao Dou, Yang Chu.Analysis of Effects on Radar Measurement Errors Due to Ship Attitude[J].Command Control \& Simulation,2011,33(6):102-105.

[9] Hongmei Li, Jianghuai Pan,Jiazhou He.Error Transfer and Sensitivity Analysis of Ship-Borne Radar Detecting[J].Journal of Data Acquisition \& Processing,2012,27(4):474-479.

[10] Deyi Sang, Jianjun Zhao, Gang Yao.The Impact on Landing Guidance Radar Precision Caused by the Movement of Aircraft Carriers[J].Chinese Journal of Ship Research,2014,9(6):8-13.

[11] "Modern fire control system.of warship " Writing Group.Modern fire control system of warships [M]. Beijing: National Defense Industry Press, 2008.

[12] Zhengcai Cao.The Commonly Used Coordinate Transformation of Stabilization Modes For Shipborne Radars[J].Radar \& ECM,2010,30(1):47-52. 